\title{
Dislocations at the interface between sapphire and GaN
}

\author{
A. Lankinen · T. Lang · S. Suihkonen - O. Svensk • \\ A. Säynätjoki $\cdot$ T. O. Tuomi · P. J. McNally · \\ M. Odnoblyudov $\cdot$ V. Bougrov $\cdot$ A. N. Danilewsky $\cdot$ \\ P. Bergman $\cdot$ R. Simon
}

(C) Springer Science+Business Media, LLC 2007

\begin{abstract}
GaN layers grown by metal organic vapour phase epitaxy on sapphire were imaged by synchrotron radiation X-ray topography. The threading dislocations could not be resolved in the topographs due to their high density, but a smaller density of about $10^{5} \mathrm{~cm}^{-2}$ defects were seen in the interface between $\mathrm{GaN}$ and sapphire by utilizing large-area back-reflection topography for the sapphire substrates. The misfit dislocation images in the topographs form a well-resolved cellular network, in which the average cell size is roughly $30 \mu \mathrm{m}$. Different cell shapes in the misfit dislocation networks are observed on different samples. Also, images of small-angle grains of
\end{abstract}

A. Lankinen $(\bowtie) \cdot$ T. Lang $\cdot$ S. Suihkonen

O. Svensk · A. Säynätjoki · T. O. Tuomi

Micro and Nanosciences Laboratory, Helsinki University of

Technology (TKK), P.O. Box 3500, Espoo 02015, Finland

e-mail: aapo.lankinen@tkk.fi

\section{P. J. McNally}

Research Institute for Networks and Communications

Engineering, Dublin City University, Dublin 9, Ireland

M. Odnoblyudov $\cdot$ V. Bougrov

OptoGaN Oy, Tietotie 3, Espoo 02150, Finland

M. Odnoblyudov · V. Bougrov

A. F. Ioffe Physico-Technical Institute, St. Petersburg 194021, Russian Federation

A. N. Danilewsky

Kristallographisches Institut, University of Freiburg, Freiburg

79104, Germany

\section{P. Bergman}

Linköping University, 58183 Linkoping, Sweden

R. Simon

ANKA, Institute for Synchrotron Radiation, Karlsruhe, Germany similar size were found in transmission section topographs of the GaN layers.

\section{Introduction}

$\mathrm{GaN}$ has been the target of concentrated research and development efforts due to its high band gap, which makes $\mathrm{GaN}$ well-suited for optoelectronic applications. The de facto standard substrate for epitaxial $\mathrm{GaN}$ has been sapphire, notwithstanding the large lattice mismatch between the two materials that makes the epitaxial techniques rather difficult. The growth of GaN on sapphire has been known to produce grains on the sub-micron scale with grain boundaries having threading (TD) and misfit (MD) dislocations [1]. In this work, large-scale structures at the interface between $\mathrm{GaN}$ and sapphire are studied.

\section{Experimental}

GaN layers having a thickness between $2-5 \mu \mathrm{m}$ were grown by metal organic vapour phase epitaxy (MOVPE) on $430 \mu \mathrm{m}$ thick (00.1) c-plane sapphire substrates utilizing the two-step or the multistep [2] nucleation layer techniques with trimethylgallium (TMGa) and $\mathrm{NH}_{3}$ as precursors. The samples selected for this study were labeled as A, B, C and D. Sample A was made using the multi-step technique and had a rather small etch pit density of about $5 \cdot 10^{7} \mathrm{~cm}^{-2}$. Sample B was grown using a more standard two-step method and had an etch pit density of over $10^{8} \mathrm{~cm}^{-2}$. Sample C contained some macroscopic dot-like defects. Sample D was also deposited with the two-step 
nucleation layer technique, but it was made with a larger ammonium flow than the other samples, which caused the $\mathrm{GaN}$ nucleation islands to grow together sooner. Sample D also had an additional p-type epilayer on top of the GaN buffer layer. Figure 1 shows a schematic structure of the samples, properties of which are listed in Table 1.

The relatively large lattice misfit between $\mathrm{GaN}$ and sapphire results in an effective $30^{\circ}$ rotation about their common hexagonal $\mathrm{c}$-axis between the two lattices, and generates MDs and strain both into the epilayer and into the substrate during the MOVPE growth. Defects in the resulting heterostructure were imaged by means of synchrotron radiation X-ray topography (SR-XRT) utilizing a continuous spectrum of wavelengths [3] at SYLAB-DESY in Hamburg and at ANKA in Karlsruhe. Topographs were recorded using large-area back-reflection and section transmission geometries [4]. In large-area back-reflection geometry the X-ray beam size was $1.5 \times 1.5 \mathrm{~mm}$ at HASYLAB-DESY and $2.0 \times 2.0 \mathrm{~mm}$ at ANKA. The $\mathrm{X}$-ray beam width in the section transmission geometry was limited in the vertical direction to $15 \mu \mathrm{m}$ using a slit made of two adjustable cylindrical tungsten bars, whereas the horizontal width of the X-ray beam was $4 \mathrm{~mm}$.

Most of the topographs of $\mathrm{GaN}$ and sapphire do not overlap on the films because of the different lattice orientations, lattice constants and crystal systems of rhombohedral sapphire and hexagonal GaN. Thus, it is possible to record images of each material individually, which can specifically be used to image the interface effects on sapphire under the relatively thick $\mathrm{GaN}$ layer. The imaging of the sapphire substrates instead of GaN layers in the large-area backreflection geometry is necessary, because the defect densities

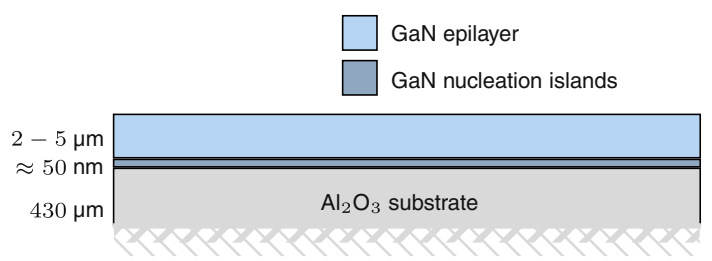

Fig. 1 Schematic drawing of the cross-section of the GaN samples. The thickness of the nucleation layer is the nominal thickness based on the volume of the nucleation islands. Sample D had an additional p-doped epilayer on top (not drawn)

Table 1 The epilayer thicknesses and growth methods of the samples

\begin{tabular}{llll}
\hline & $\begin{array}{l}\text { GaN thickness } \\
(\mu \mathrm{m})\end{array}$ & $\begin{array}{l}\text { Additional } \\
\text { epilayers }\end{array}$ & $\begin{array}{l}\text { Growth } \\
\text { method }\end{array}$ \\
\hline Sample A & 5 & - & multistep \\
Sample B & 3.5 & - & two-step \\
Sample C & 2 & - & two-step \\
Sample D & 2 & $1.35 \mu \mathrm{m}$ p-doped & two-step \\
\hline
\end{tabular}

of the GaN layers are too large for observation of individual dislocations using large-area SR-XRT. In this work, the hexagonal coordinate system is used for the rhombohedral lattice of sapphire in order to obtain the same coordinate system for the GaN epilayers and sapphire substrates. In addition to SR-XRT images, atomic force micrographs (AFM) of the etched GaN surfaces were recorded in order to obtain etch pit density data on GaN.

\section{Results and discussion}

Overall TD dislocation densities were calculated from AFM images. Two AFM micrographs of sample A are shown in Fig. 2. Two distinct types of etch pits were observed. Figure 2 a shows only small circular etch pits, the density of which is about $5 \cdot 10^{7} \mathrm{~cm}^{-2}$. The small etch pits are most likely endpoints of pure edge threading dislocations [5]. In addition to the small circular etch pits Fig. $2 b$ shows two large etch pits, which are clearly hexagonal and appear to have a density of less than $10^{5} \mathrm{~cm}^{-2}$. The large hexagonal etch pits are believed to be generated by screw dislocation or mixed dislocation endpoints on the GaN surface [5]. It was also noticed that the TD endpoints tend to form clusters and to line up in the AFM micrographs.

Figure 3 shows a $1 \overline{1} .14$ large-area back-reflection topograph of the sapphire substrate beneath the $5 \mu \mathrm{m}$ thick GaN layer in sample A. The topograph shows a wellresolved irregular cellular network having a cell size of roughly $30 \mu \mathrm{m}$ in diameter. Several large-area backreflection topographs of bare sapphire substrates were recorded for reference, but those are not shown in this work, as they only had images of characteristic threading dislocations in the sapphire substrates. According to the kinematical theory of diffraction the black images on the grey sapphire background in Fig. 3 indicate either defects or high local strain fields. The defects in the sapphire are

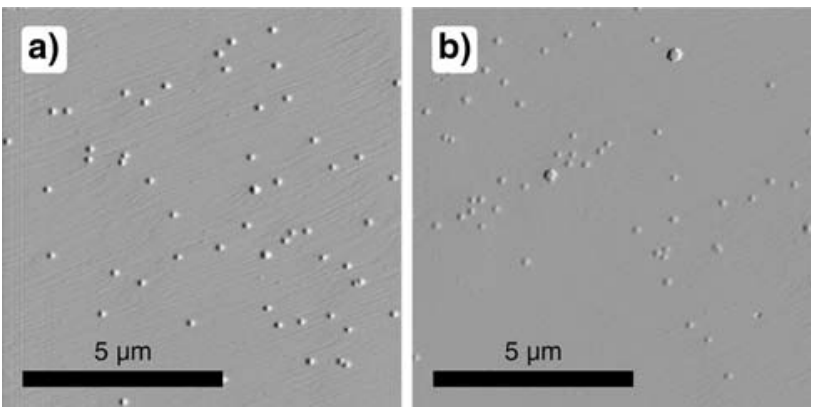

Fig. 2 Deflection mode AFM-images of etched GaN surface showing (a) only small circular etch pits and (b) also some larger hexagonal etch pits. The hexagonal etch pits are believed to be formed at screw dislocation exit points. Image size is $10 \times 10 \mu \mathrm{m}$ 


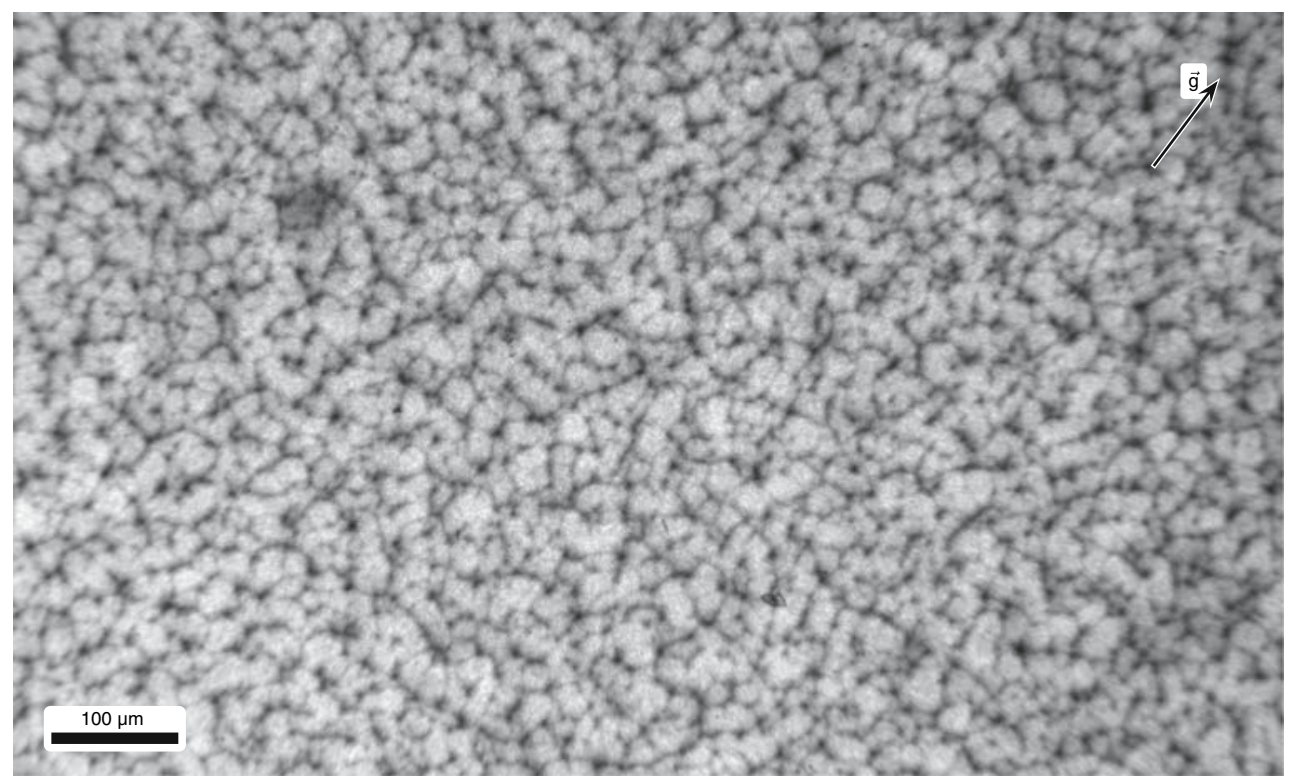

Fig. $31 \overline{1} .14$ large-area back-reflection topograph of the sapphire lattice of sample A showing the strain from the MD network in the sapphire$\mathrm{GaN}$ interface. Diffraction vector $\vec{g}$ projection is in the top right corner. Image size is $1000 \times 600 \mu \mathrm{m}$

obviously caused by the relaxation of strain produced by the $\mathrm{GaN}$ epilayer, and thus the defects are likely on the surface of the sapphire substrate. The image of the cellular network in the topograph consists of individual black dots and thin lines connecting the dots. The thin lines may actually consist of defect dots lined up similarly to the lined-up dislocation endpoints in Figs. $2 \mathrm{a}$ and $\mathrm{b}$, but the resolution of the SR-XRT is not sufficient to ascertain this. The number of the individual defect images in the topograph is roughly $10^{5} \mathrm{~cm}^{-2}$, excluding the possible finestructure of the thin lines. The observed cellular network of defects in sapphire probably also extends from the interface to at least some distance into the GaN epilayer. However, it is not known whether the clustering and lining-up of the etch pits in the AFM micrographs of Fig. 2 is connected to the cellular network of defects observed in sapphire by SRXRT, because the TD endpoints on the GaN surface may very well have a different pattern than the dislocations at the sapphire-GaN interface.

Figure 4 shows large-area back-reflection topographs of the sapphire substrate surface of the samples B, C and D. Figure 4a shows a topograph of sample B having a GaN epilayer with a large TD density of over $10^{8} \mathrm{~cm}^{-2}$. The defect network images in the topograph have noticeably smaller cell size and more signs of strain than in Fig. 3. The topograph in Fig. $4 \mathrm{~b}$ is recorded from sample D and shows a peculiar cell structure consisting of irregular hexagons having a defect dot in the centre. Similar hexagonal cells with a central defect dot were also observed in Sample A, albeit there are much fewer such cells and they are more irregular in the topograph of sample A in Fig. 3.
The size of the cells in sample D is approximately $30 \mu \mathrm{m}$ like those of sample A. Figure $4 \mathrm{c}$ from sample $\mathrm{C}$ shows topography images of strain and defects in sapphire caused by a macroscopic defect in the GaN epilayer. This large defect image having a diameter of $100 \mu \mathrm{m}$ is clearly visible in the upper part of the topograph. Notwithstanding the large defect image in Fig. 4c, the overall contrast in the topograph of sample $\mathrm{C}$ is rather weak, which indicates that the $\mathrm{GaN}$ epilayer of the sample $\mathrm{C}$ imposes only a minute strain onto the sapphire substrate.

$\overline{2} 0.1$ and 20.1 transmission section topographs of the $\mathrm{GaN}$ epilayer and $\overline{23} .4$ topographs of the sapphire substrate recorded from sample A in a single exposure are shown in Fig. 5. The GaN epilayer images show small-angle boundaries between the GaN cells. However, the GaN epilayer line is not fuzzy or broadened in the topograph despite the misfit dislocations, which indicates a relatively good crystal quality. More specifically, the GaN epilayer images in Fig. 5 show a sharper contrast than GaN epilayers topographed in comparable previous works $[6,7]$, where section topograph images of the $\mathrm{GaN}$ epilayers were significantly broadened. The cellular network of small-angle grain boundaries in the sapphire layer of Fig. 3 is believed to be replicated in the GaN layer, but unfortunately the overall dislocation density, $5 \cdot 10^{7} \mathrm{~cm}^{-3}$, of the $\mathrm{GaN}$ epilayer is too large for resolving individual dislocations in large-area SR-XRT. However, it is very likely that the small-angle grain boundaries seen on sapphire extend into the GaN layer, which is also supported by the fact that the small-angle grains in the GaN transmission section topographs of Fig. 5 are of the same size as the cell 

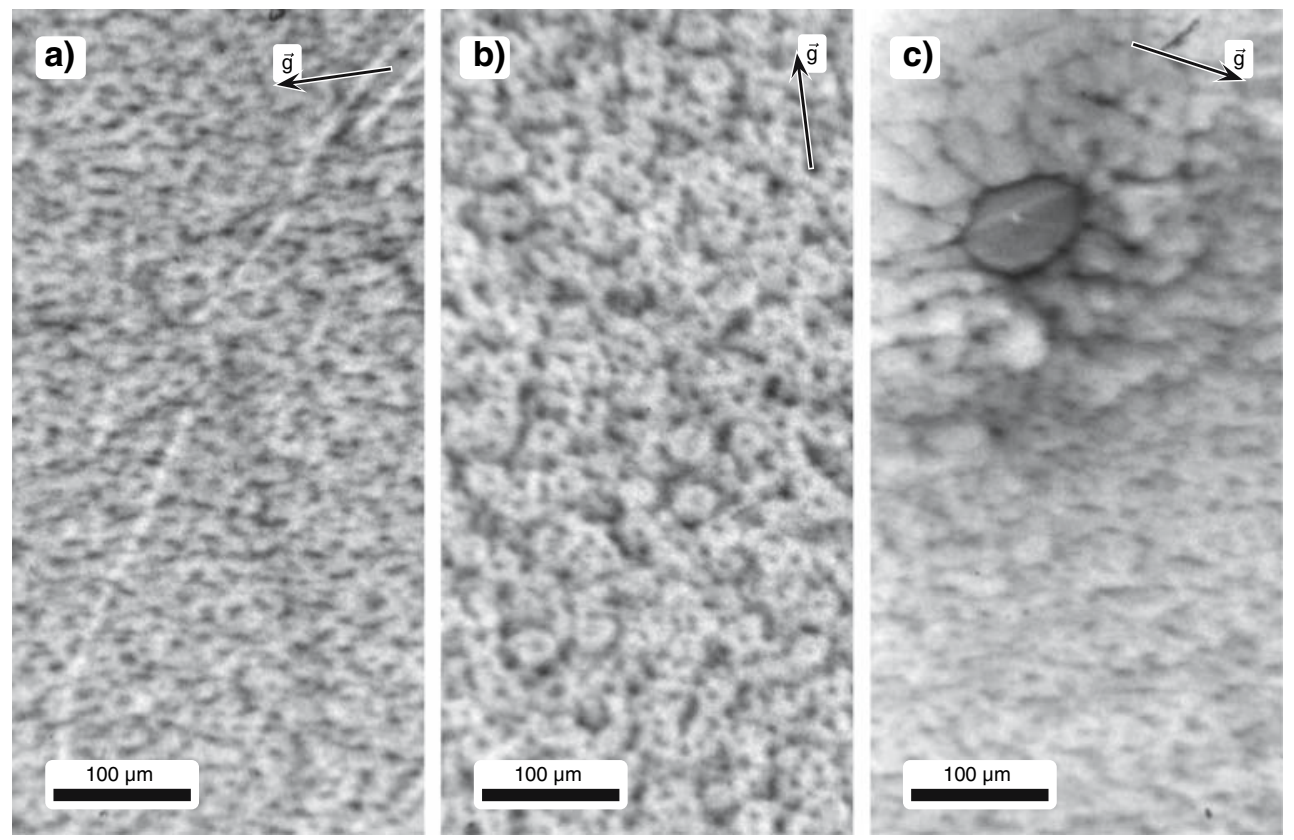

Fig. 4 Large-area back-reflection topographs (a) 22.14 of sample B, (b) 03.18 of sample D, and (c) 03.18 of sample C showing the strain from the MD network in the sapphire-GaN interface. Diffraction vector $\vec{g}$ projections are shown in the topographs. Image size is $300 \times 600 \mu \mathrm{m}$
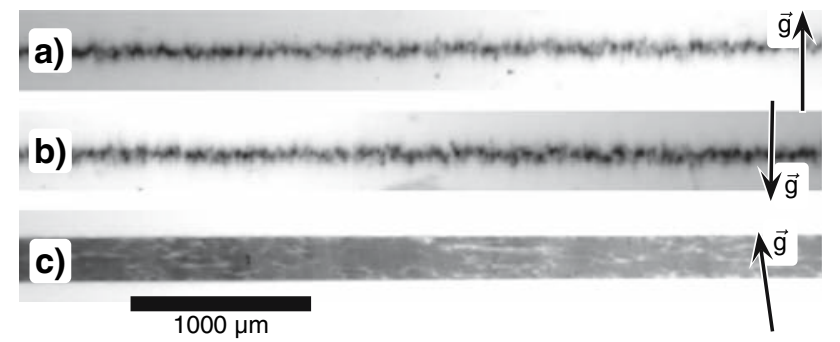

Fig. 5 (a) $\overline{2} 0.1$ and (b) 20.1 transmission section topographs of the $\mathrm{GaN}$ epilayer and (c) $\overline{2} \overline{3} .4$ of the sapphire substrate of sample A. The topographs were recorded simultaneously on the same film. The distance between adjacent small angle boundaries in the GaN epilayer is $30-40 \mu \mathrm{m}$. Diffraction vector $\vec{g}$ projections are shown in the topographs

images in the large-area back-reflection sapphire topograph of Fig. 3. Even though the GaN epilayer in sample A is not strictly speaking monocrystalline as there clearly are features possessing many grain boundaries, the misalignment between the small-angle grains appears to be so small that the grains are practically in the same orientation. Thus, sample A can be considered to have a monocrystalline GaN epilayer having about $5 \cdot 10^{7}$ dislocations $/ \mathrm{cm}^{2}$, some of which are dislocations forming a cellular structure of small-angle grain boundaries.

Figure 6 shows transmission section topographs of samples A, B, C and D. The diffraction vectors $\vec{g}$ in these topographs are perpendicular to the c-axes of the lattices. Recording of such topographs was made possible by rotating the samples $6^{\circ}$ about an axis coincident with the plane section of the sample surface and the diffracting plane. Thus, the Bragg angle for these reflections is $\theta_{B}=$ $6^{\circ}$. For this geometry distances in the c-axis direction are foreshoretened by $\tan \left(2 \theta_{B}\right) / \cos \left(\theta_{B}\right) \approx 0.21$, whereas distances measured in directions perpendicular to c-axis remain the same. This effectively makes the images of $430 \mu \mathrm{m}$ thick sapphire substrates in the topographs of Fig. 6 to appear only about $100 \mu \mathrm{m}$ thick.

The topograph in Fig. 6a of sample A has the GaN epilayer image overlaid with the sapphire substrate image, because the GaN epilayer in sample A happened to have a small tilt angle (calculated value $<0.05^{\circ}$ ) between the substrate and the epilayer lattice c-axes in such a direction that the GaN epilayer image was rotated exactly on top of the sapphire substrate image. However, based on topographs of Fig. $5 b$ and $c$ it can be observed that the GaN epilayer in sample A has the best quality of the samples, because it has the thinnest epilayer image compared to the substrate image thickness in the section topographs, even though sample A has the thickest $\mathrm{GaN}$ epilayer. The comparison between the thicknesses of the $\mathrm{GaN}$ epilayer image in Fig. $5 b$ and the sapphire substrate image in Fig. $5 \mathrm{c}$ is possible, because the topographs in these figures are located so close on the film that the geometrical projections for these two topographs are practically the same.

The GaN epilayer image of sample B in Fig. $6 b$ has translated slightly above the substrate image and thus is completely visible in the topograph. The epilayer in sample 

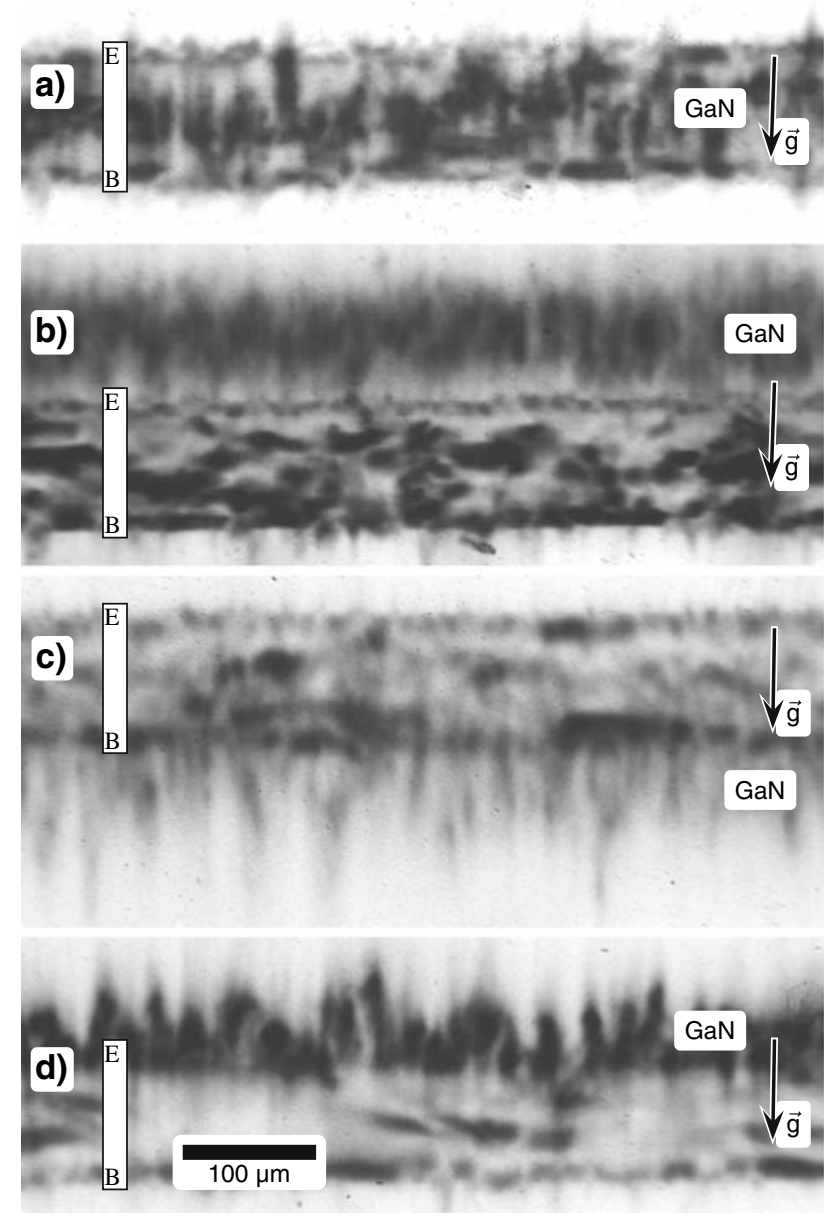

Fig. 6 Transmission section topographs (a) $\overline{1} \overline{1} .0(\mathrm{GaN}) \quad 01.0$ (sapphire) of sample A, (b) $10.0(\mathrm{GaN}) \overline{1} \overline{1} .0$ (sapphire) of sample $\mathrm{B}$, (c) $\overline{1} 1.0(\mathrm{GaN}) 01.0$ (sapphire) of sample C, and (d) 11.0 $(\mathrm{GaN}) 0 \overline{1} .0$ (sapphire) of sample D. The vertical placement of the $\mathrm{GaN}$ layer is indicated in the topographs, as the placement varies according to a potential small tilt between the substrate and the epilayer lattices. White solid bars denote the sapphire substrate orientation having the episide of the substrate marked as $\mathrm{E}$ and backside as B. The diffraction vectors $\vec{g}$ are perpendicular to the hexagonal c-axes and their projections on the films are shown in the topographs

B is seen to be of poorer quality than that of sample A, because the diffraction image of the sample B epilayer in Fig. $6 \mathrm{~b}$ is much fuzzier than that of sample A in Figs. 5a and $b$. The sharp boundaries between the defected areas disappear, as the images of the dislocations between the small-angle boundaries are so numerous that their combined intensity is visible as a grey and indistinct intensity distribution rather than as sharp-edged contrast changes between the grains. Fig. $6 \mathrm{c}$ shows how the $\mathrm{GaN}$ epilayer in sample $\mathrm{C}$ has such a low crystal quality that the epilayer grain images are elongated and very faint. The small-angle grain boundaries in the section topograph of sample D in Fig. $6 c$ are expectionally clear and easily distinguishable.
The grain sizes appear to be varying between $20-40 \mu \mathrm{m}$, which is in a good agreement with the sapphire defect cell sizes of the same sample visible in the topograph of Fig. $4 \mathrm{~b}$, taking into account the $15 \mu \mathrm{m}$ wide section X-ray beam bisecting an individual grain at a random position. Also, clear images of the small-angle boundary defects indicate a rather good quality in the epitaxial layer, which is further suggested by the fact that the image of the $\mathrm{GaN}$ epilayer is almost as thin as that of sample A in Fig. 5b. The GaN epilayer in sample D appears to be exactly in the same orientation as the sapphire substrate lattice, in contrast to samples A, B and C, which all have a small tilt angle between the substrate and epilayer lattice c-axes. Also, the substrate image of sample D in Fig. 6d displays fewer defects than the substrate images of samples A, B and $\mathrm{C}$ in Figs. $6 \mathrm{a}, \mathrm{b}$ and $\mathrm{c}$.

An interesting aspect of the obtained results is the large size of the MD network cells on the sapphire substrates, and also the large size of the corresponding grains in the GaN epilayers. The diameter of the largest nucleation islands in sample A has probably been $2-5 \mu \mathrm{m}$ before the start of the two dimensional GaN growth, and the overall nucleation island density has likely been approximately $10^{7} \mathrm{~cm}^{-2}$ [8]. Thus, if the MD network cells are formed by nucleation islands growing together, one such cell should consist of roughly $10^{2}$ nucleation islands in the case of sample A. Earlier studies have shown that the GaN growth is dominated by the originally larger nucleation islands, and that the small nucleation islands are suppressed until the start of the two dimensional growth [8], but it is not known whether this effect could extend to structures as large as the MD network cells observed in this work. However, the similar size of the MD network cells between samples A and D despite the use of different growth techniques suggests that the MD network cell sizes or the $\mathrm{GaN}$ small-angle grain sizes are not connected to the $\mathrm{GaN}$ nucleation island sizes.

Another explanation for the observed $30 \mu \mathrm{m}$ average size of the MD network cells in samples A and D could simply be a misfit production mechanism caused by the large misfit between sapphire and $\mathrm{GaN}$, in which case the cellular network should be very similar in all good-quality epitaxial GaN on sapphire. However, the average distance between MDs at the interface between $\mathrm{GaN}$ and sapphire is just $2 \mathrm{~nm}[9,10]$. Even though these interfacial MDs in $\mathrm{GaN}$ are shown to be very regularly distributed [9], it is difficult to think of a general MD production mechanism producing large misfit cells of $30 \mu \mathrm{m}$ in diameter given the very dense MD network at the interface. Obviously, the dense MD network at the interface is not visible in any way in the topographs studied in this work due to the large difference in the scales involved. For these reasons, it was unfortunately not possible to ascertain the validity of either 
of the discussed hypotheses for the origin of the large cellular MD networks observed within this work.

\section{Conclusions}

Images of strain caused by different cellular networks of MDs at the interface between sapphire and GaN were imaged using SR-XRT in the large-area back-reflection mode. Small-angle grain boundaries in the GaN epilayers were observed using SR-XRT in the transmission section mode. Structures having regular cellular network with cell size of roughly $30 \mu \mathrm{m}$ were observed in two samples. Samples having a GaN layer with considerably more defects had more irregular cellular MD network structures and also smaller MD network cell sizes. The size of the small-angle grains in the GaN epilayers was observed to be the same as the MD defect cell sizes in the sapphire substrates. The observed small-angle grains and MD network cells had considerably larger diameters than the GaN nucleation islands at the start of the growth process are known to have.

Acknowledgements This work was supported by the European Community - Research Infrastructure Action under the FP6 "Structuring the European Research Area" Programme (through the
Integrated Infrastructure Initiative "Integrating Activity on Synchrotron and Free Electron Laser Science'.) M. Odnoblyudov and V. Bougrov acknowledge support by RFBR and Dynasty Foundation. Also, we would like to thank Dr. C. Paulmann for his assistance at HASYLAB beamline F1 Topography.

\section{References}

1. X.H. Wu, P. Fini, E.J. Tarsa, B. Heying, S. Keller, U.K. Mishra, S.P. DenBaars, J.S. Speck, J. Cryst. Growth 189/190, 231 (1998)

2. T. Lang, M. Odnoblyudov, V. Bougrov, M. Sopanen, J. Cryst. Growth 277, 64 (2005)

3. T. Tuomi, K. Naukkarinen, P. Rabe, Phys. Stat. Sol. (A) 25, 93 (1974)

4. T. Tuomi, J. Synchrotron Radiation 9, 174 (2002)

5. K. Shiojima, J. Vacuum Sci. \& Tech. B: Microelectronics and Nanometer Structures 18, 37 (2000)

6. P.J. McNally, T. Tuomi, D. Lowney, K. Jacobs, A.N. Danilewsky, R. Rantamäki, M. O’Hare, L. Considine, Phys. Stat. Sol. (A) 185, 373 (2001)

7. W.M. Chen, P.J. McNally, K. Jacobs, T. Tuomi, A.N. Danilewsky, Z.R. Zytkiewicz, D. Lowney, J. Kanatharana, L. Knuuttila, J. Riikonen, J. Cryst. Growth 243, 94 (2002)

8. T. Lang, M. Odnoblyudov, V. Bougrov, S. Suihkonen, M. Sopanen, H. Lipsanen, J. Cryst. Growth 292, 26 (2006)

9. S. Kaiser, H. Preis, W. Gebhardt, O. Ambacher, H. Angerer, M. Stutzmann, A. Rosenauer, D. Gerthsen, Jpn. J. Appl. Phys. 37, 84 (1998)

10. J. Jasinski, Z. Liliental-Weber, J. El. Mat. 31, 429 (2002) 